\title{
Principios, progresividad y factibilidades de la recuperación de "plusvalías" urbanas en el Chile actual'
}

\section{Principles, progressivity and feasibility of the recovery of "capital gains" urban in Chile today}

\author{
Carolina Páramo Lopera² 기 y Ernesto López-Morales³
}

\begin{abstract}
RESUMEN
La urbanización en Chile, desarrollada por agentes privados, se caracteriza por ejecutar el máximo aprovechamiento normativo y capitalizar el acceso a bienes públicos. Las rentas de suelo excedentes (plusvalías urbanas), de origen colectivo, no retornan a la comunidad al no existir instrumentos diseñados para recuperarlas y redistribuirlas. Recientes avances en el marco jurídico de la planificación urbana en el país se centran en exigir al sector inmobiliario la internalización de externalidades producidas por densificación, y en capturar parcialmente la valorización generada por recalificación de suelo rural. Sin embargo, la plusvalía producida por inversión en obras públicas, que es principalmente aprovechada por los agentes inmobiliarios privados, no es objeto de recuperación. Este artículo aborda aspectos teóricos de la formación de las rentas del suelo urbano, aportando evidencia empírica de fuente secundaria de la plusvalía generada por la red de Metro de Santiago de Chile, para posteriormente analizar las nuevas leyes, enfatizando su capacidad de recuperar y redistribuir la plusvalía urbana, pero anticipando sus efectos regresivos derivados de su aplicación territorial.
\end{abstract}

Palabras clave: Recuperación de plusvalias urbanas, desarrollo inmobiliario, políticas urbanas, América Latina.

\begin{abstract}
Privately-led real estate urbanization in Chile is characterized by the highest land use. While private developers internalize the positive effects generated by public amenities, the increased land value, which is generated by public efforts, is not recovered by, nor redistributed to, the community, as in Chile there are no planning instruments specifically aimed to make those two goals possible. However, some recent legal and planning adjustments seek the internalization of the externalities produced by urban densification by private agents, and also currently the state can partially capture land valuation generated by rural-to-urban rezoning. However, still land value generated by public infrastructure is hitherto not captured by the state. This article explores theoretical aspects of land value formation, seeking to provide empirical evidence about the effect produced on this valuation by the Metro network in Santiago, Chile. Furthermore, the paper analyses the new legal framework in the country, addressing its actual capacity to capture and redistribute land value, and anticipating regressive effects derived from its application.
\end{abstract}

Key words: Land value capture, real estate, urban policies, Latin America. 


\section{Introducción}

En las últimas décadas, las capitales latinoamericanas han experimentado procesos de inversión pública urbana, con resultados inequitativos evidenciados en la concentración de la dotación de esos servicios en espacios urbanos centrales y de altos ingresos. Por otra parte, han existido carencias de inversión en infraestructura básica en zonas desventajadas, crónicamente carentes de acceso a estas mejoras. La más reciente tendencia a la densificación vía construcción en altura ha sido concomitante con este desarrollo inequitativo; esta tendencia se observa en predios que se valorizan en función de su localización central y acceso a infraestructura pública, y cuya normativa urbana permite altos aprovechamientos y exige mínimos aportes urbanos en contraprestación (Smolka, 2014). El resultado han sido mercados inmobiliarios masivos y expansivos, que permiten capitalizar altas rentas de suelo prácticamente sólo al desarrollador y, por su extensión, a los inversionistas privados que los financian, y en bastante menor medida al Estado, cuyas capacidades administrativas territoriales se ven a menudo sobrepasadas (Jaramillo, 2008).

La racionalidad de esto es bastante clara: el Estado invierte por mejorar la accesibilidad o dotación de bienes públicos de un determinado lugar. Sin embargo, correspondería que quienes más aprovechan esas ventajas, retribuyan total o al menos parcialmente esa inversión pública. Entre los países latinoamericano que se destacan por articular principios relacionados con esta "recuperación" de la plusvalía urbana por parte del Estado para beneficio comunitario, y que cuentan con legislaciones explicitas para ello, llegando en algunos casos a disposiciones constitucionales y leyes específicas, se cuentan Colombia ${ }^{4}$, Brasil $^{5}$, Ecuador ${ }^{6}$ y Uruguay ${ }^{7}$ como los casos más patentes. Allí, la recuperación de las plusvalías (que, en exacto rigor, y como se verá más adelante, son "rentas de suelo") supone un ejercicio de restitución a la comunidad y redistribución equitativa de las valorizaciones excedentes, buscando restablecer "[...] la equidad rota por la adjudicación imperfecta, no transparente, de beneficios y costos del desarrollo urbano" (Cáceres \& Sabatini, 2001: 122).

No obstante la abundante legislación respecto de la recuperación de plusvalías urbanas en América Latina, su definición es difusa y su implementación y fiscalización, débil (Smolka, 2014; Maldonado, 2007; Furtado, 1997). Aun así, en los últimos 20 años, gobiernos nacionales de países sudamericanos han asumido la tarea de robustecer el instrumental de ordenamiento territorial y de fortalecer las competencias administrativas locales con el fin de promover la autofinanciación de la política urbana municipal por medio de la aplicación de instrumentos de recuperación de plusvalía.

\footnotetext{
La Constitución Política de Colombia de 1991 define la función social y ecológica de la propiedad, la prevalencia del interés general sobre el colectivo y la participación de las entidades públicas en las plusvalias que se generen por sus actuaciones. La Ley 388 de 1997 (República de Colombia) define el instrumento de Participación en Plusvalía.

5 La Ley Federal N 10.257 "Estatuto de la Ciudad" (Gobierno Federal de Brasil), incluye una gama de instrumentos de recuperación de plusvalía como son el Otorgamiento Oneroso de Derechos de Construcción y la Subasta de Derechos Adicionales de Construcción (CEPAC's).

6 Ley Orgánica de Ordenamiento Territorial, Uso y Gestión del Suelo (Gobierno del Ecuador, 2016). Con el objetivo de recuperar plusvalías para el desarrollo, la ley dispone de instrumentos de gestión donde se incluyen los dedicados a la distribución equitativa de cargas y beneficios, a la regulación del mercado del suelo y al financiamiento del desarrollo urbano.

La Ley No 18.308 "Ley de Ordenamiento Territorial y Desarrollo Sostenible" (Republica del Uruguay) en su Artículo 46 establece la recuperación como mínimo del $15 \%$ del incremento derivado de las acciones públicas.
} 
En el otro extremo se encuentra Chile, como un caso donde se ha apostado por el fortalecimiento del sector inmobiliario, dominante de la actividad de la construcción, y pilar de la estrategia económica de crecimiento de PIB nacional y regional. No se ha establecido en este país, sin embargo, la articulación del poderoso sector inmobiliario privado con una legislación de desarrollo urbano acorde, que contemple aplicación de instrumentos de planificación y gestión, y menos de recuperación de plusvalía. Sólo recientemente en 2016, se ha promulgado una Ley de Aportes $^{8}$, los cuales se orientan a reinvertirse en las mismas comunas desde donde se extrae el cobro de la compensación.

El presente artículo se focaliza precisamente en el caso de Santiago de Chile, una ciudad en la que el Estado, a la fecha (2019), no ve redistribuir la plusvalía urbana que genera con la materialización de la obra pública urbana. Trivelli (2015:46) afirma que "las plusvalías han tenido históricamente un tratamiento tributario muy benévolo" en Chile, situación agravada por las pocas iniciativas implementadas para su recuperación, que además descansan en numerosos criterios de exención para su pago. Por su parte, una serie de estudios (Agostini \& Palmucci, 2008; Transsa \& Atisba, 2014; Vega, 2017; López-Morales et al., 2019) confirman que la expansión de la red de Metro de Santiago es el principal hecho generador de plusvalía capitalizada por agentes privados, sin posibilidad de retorno a la comunidad, al no existir en la actualidad, instrumentos dedicados expresamente a su recuperación. Asimismo, municipios que acometen acciones de planificación de tipo normativo, ventajosas para aumentar el volumen de la construcción, son también generadores de plusvalía urbana a ser capturada por las inmobiliarias (López-Morales et al., 2019). Muchas de las comunas de Santiago compiten por atraer densificación y por ende son donde agentes inmobiliarios aprovechan al máximo la normativa otorgada; tales comunas son políticamente independientes en la fijación de sus normas urbanas (López-Morales, Meza \& Gasic, 2014; Vicuña, 2018).

Los incrementos en las rentas del suelo se originan gracias a que la función reguladora estatal otorga aprovechamientos a cada porción de suelo, aportando valor en función de lo que en éste puede edificarse, mientras que proyecta y dota a la ciudad de infraestructuras que garantizan la accesibilidad desde y hacia ese suelo. Al no existir instrumentos específicamente orientados a su recuperación, al final del día, las rentas urbanas incrementadas pasan directamente a manos de quienes detentan la propiedad jurídica del suelo y, más importante aún, de quienes poseen el capital para invertir y los medios de producción para lograr el máximo aprovechamiento de las ventajas instaladas (López-Morales, 2015), es decir los conglomerados o asociaciones financiero-inmobiliarias (Wainer et al., 2016). Se configura así, una tendencia a la concentración en algunas pocas empresas, no de la propiedad del suelo, sino que de la capacidad de aprovechamiento del potencial de construcción sobre ese suelo y, con esto, una tendencia al monopolio de la captura de la plusvalía urbana.

Como respuesta a la patente concentración de las rentas del suelo, los instrumentos de recuperación de plusvalía de orden fiscal o regulatorio (Amborski \& Smolka, 2003) son aplicables en diferentes momentos y persiguen objetivos distintos en el marco de la recuperación de plusvalías urbanas. Asi, los instrumentos fiscales "requieren alguna forma de impuesto o contribución a ser pagada por el propietario de la tierra" mientras que "los instrumentos regulatorios llevan a alguna forma de beneficio público, también pagado por el propietario con cargo al incremento en el va-

Ley N²0.958 "Establece un sistema de aportes al espacio público". Diario Oficial de la República de Chile del 15 de Octubre de 2016 
lor del suelo", por ejemplo, a través de algún tipo de contribución en especie que se traduce en cesiones de suelo edificable o en obras de dotación urbana en sitio. Blanco et al. (2016) señalan que los mecanismos como la "Participación en Plusvalía" (Colombia), el "Otorgamiento Oneroso" (Brasil) y las "Contribuciones por Mejoras" en sus diferentes versiones, son considerados como instrumentos fiscales aplicados específicamente para capturar la valorización del suelo generada por distintas acciones de desarrollo urbano. Para estos autores, los instrumentos fiscales tienen la capacidad de recuperar las plusvalías en etapas anteriores a la urbanización, lo que los convierte en mecanismos de recuperación ex ante, mientras que los instrumentos regulatorios de gestión asociada, como son el reajuste de tierras y las obligaciones urbanísticas, implican en la distribución de cargas y beneficios o compensaciones asumidas como cesiones de suelo, una recuperación de plusvalía ex post.

La identificación de qué instrumento o mecanismo emplear en la articulación entre la financiación local del desarrollo urbano, y el logro de los objetivos públicos mencionados, pasa por reconocer las condiciones más favorables para la implementación de acuerdo con cada mecanismo. De esta manera, los instrumentos de recuperación de plusvalía que son diversos, se relacionan entre sí y actúan en diferentes escalas de la planificación y la gestión urbana, involucrando procesos, tiempos y actores diferentes. Asimismo, aunque se reconoce la necesidad de hacer partícipe al Estado de la recuperación de las rentas de suelo que genera, el cobro de plusvalías también encuentra oposición desde la disposición negativa, generalizada e histórica de la población a pagar impuestos. Arriagada \& Simioni (2002:11) destacan que además, las rentas adicionales del suelo son consideradas como "beneficios o derechos del propietario", cuyo aprovechamiento y disfrute en el caso chileno, está fuertemente resguardado por el derecho de propiedad consagrado en la Constitución Política de 1980.

Ahora, es importante reconocer que una eventual implementación de una política de recuperación de plusvalías urbanas en Chile no parte de cero. Los antecedentes históricos remiten a cuatro momentos en el tiempo:

1. Una implementación previa, aunque inconstante, de mecanismos de recuperación de plusvalía, con los cuales se han logrado financiar proyectos urbanos de escala municipal. El caso del financiamiento del Parque Bustamante en Santiago o pasajes peatonales en la comuna de Providencia, durante la primera mitad del Siglo XX, son buenos ejemplos.

\footnotetext{
En Chile, desde principios del Siglo XX, la "Contribución de Mejoras" logró la financiación compartida de algunas obras de la ciudad, aunque no establecerse como legislación permanente a futuro. Un ejemplo es la Ley No. 4180 de 1927 que "Aprueba Proyecto de Pavimentación de Santiago" que establecía el pago de contribuciones por parte de los propietarios de predios colindantes a las obras de pavimentación. Otro ejemplo es el proyecto del Parque Bustamante (1929) y el Programa Municipal de Pasajes Peatonales, comuna de Providencia (1976). El primero empleó un esquema de contribuciones diferenciadas aplicadas a cada predio y que decrecían en su monto en función de la proximidad al proyecto. El segundo, fue concebido como parte de las acciones del Plan Regulador Comunal de Providencia de 1976, bajo la estrategia de permeabilización de las manzanas y consistió en un ejercicio de gestión urbana donde los cambios en las normas de uso del suelo, fueron negociados "caso a caso con los propietarios interesados en llevar a adelante proyectos inmobiliarios" (Cáceres \& Sabatini, 2001:139). La negociación consistió en el ofrecimiento por parte de la Municipalidad, de cinco Metros cuadrados para oficinas y/o vivienda, por cada m2 de suelo privado cedido para construir pasajes peatonales. Su implementación no requirió de la creación y pago de contribuciones e impuestos adicionales, ni de la expropiación de terrenos. Estos casos muestran que aun cuando la legislación urbana de mayor jerarquía no acompañe estas acciones, la gestión local puede lograr activar ejercicios de financiación público - privada, sentando el precedente para formulaciones e implementaciones futuras.
} 
2. Actualmente, existe el impuesto territorial o contribución de bienes raíces. Esta herramienta tributaria no es considerada como recuperadora de plusvalía urbana, aunque su perfeccionamiento y proyección hacia un impuesto robusto de base suelo podría convertirla en un instrumento para recuperar y movilizar plusvalías urbanas (Orrego, 2017).

3. En los últimos tres años se han promulgado leyes (Ley $N^{\circ} 21.078$ "Sobre transparencia del mercado del suelo e impuesto al aumento de valor por ampliación del límite urbano" y Ley N²0.958 "Establece un sistema de aportes al espacio público", 2016) y políticas públicas de temática urbana (Política Nacional de Desarrollo Urbano, 2014 y Propuesta para una política de suelo para la integración social urbana, 2015), facilitando la base para una futura implementación e instalando el debate sobre la recuperación de "plusvalías" con alcance redistributivo.

4. Un creciente interés académico y político en el estudio del proceso de formación y recuperación de la "plusvalía" urbana.

El presente artículo analiza en profundidad los principios y factibilidades asociados al tercer punto arriba mencionado, es decir, la aplicación de instrumentos de recuperación de renta de suelo para el contexto actual chileno. Los objetivos del artículo son: primero, clarificar conceptualmente la renta de suelo (o plusvalía urbana) generada en la ciudad; segundo, analizar la formación de estas rentas en Santiago y cómo esta formación se correlaciona con la existencia de Metro y regulaciones de suelo y construcción; tercero, proveer de un breve estado de situación de la recuperación de rentas de suelo o plusvalías urbanas en América Latina y Chile; cuarto, analizar críticamente los instrumentos actuales orientados a esta recuperación en Chile, concluyéndose en su carácter regresivo, al potenciar la valorización in-situ y desarrollo desigual de la ciudad. Finalmente, la sección de conclusiones aborda nuevamente la discusión teórica del asunto a la luz de la información recopilada. La sección siguiente explica la metodología ocupada en este estudio.

\section{Metodología}

El presente artículo resume una investigación exploratoria, empírica y documental realizada para el caso chileno. La investigación se basó en una extensa revisión de la literatura existente en torno a la formación, recuperación y redistribución de la renta del suelo (comúnmente denominada "plusvalía urbana", para efectos de este artículo se utilizará esta denominación). Cabe aclarar, que pese a ser un tema instalado en el debate público y político y que requiere mayor profundidad investigativa, a la fecha se cuenta con un reducido número de estudios que proporcionan aportes cuantitativos sobre la generación de plusvalías urbana en Santiago de Chile. De tal forma, la información y tipo de análisis desplegado en este estudio es de tipo conceptual y cualitativo, y en menor medida cuantitativo. En el marco de lo descrito, fueron realizadas 13 entrevistas a expertos chilenos e internacionales, y se revisaron en detalle siete investigaciones realizadas entre 2014 y 2017, de las cuales se analizan los resultados de sólo cinco, por ser éstas las que se encuentran focalizadas en el estudio de la generación de plusvalía por el anuncio y construcción del Metro de Santiago. Las investigaciones consultadas, sus objetivos, metodología y resultados se resumen en el Cuadro $N^{\circ} 1$. 


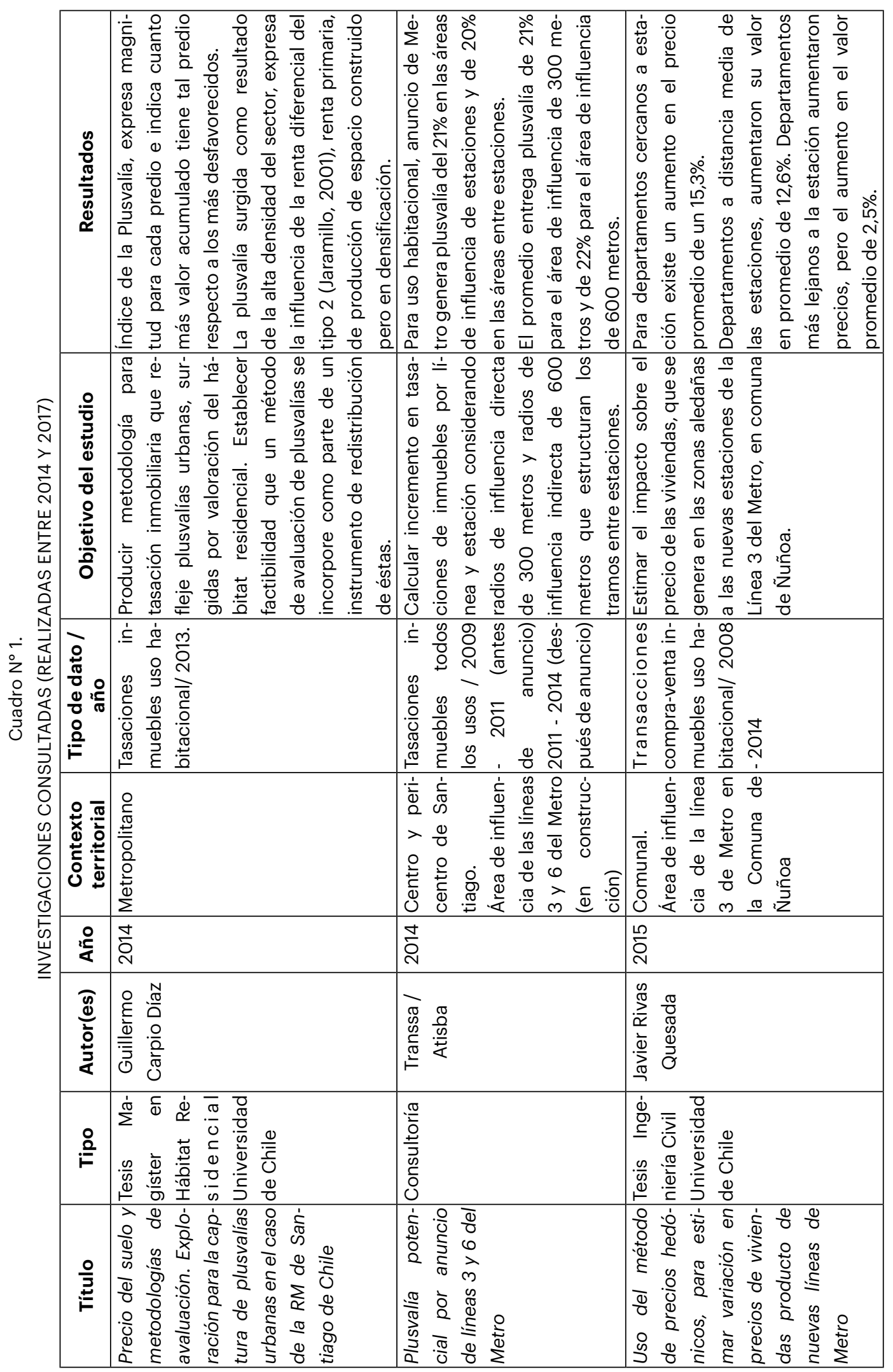




\begin{tabular}{|c|c|c|}
\hline 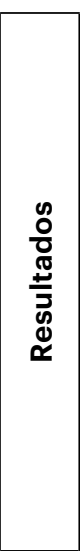 & 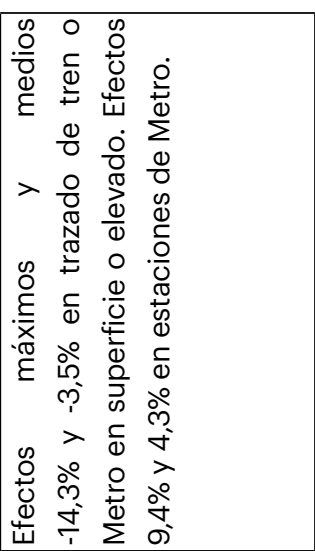 & 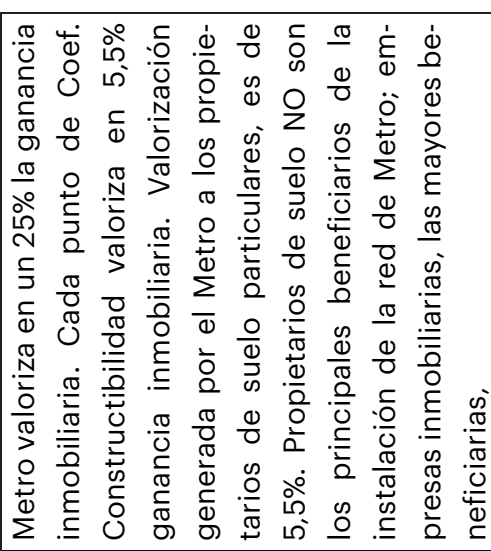 \\
\hline 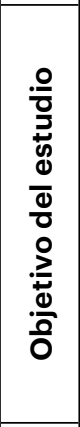 & 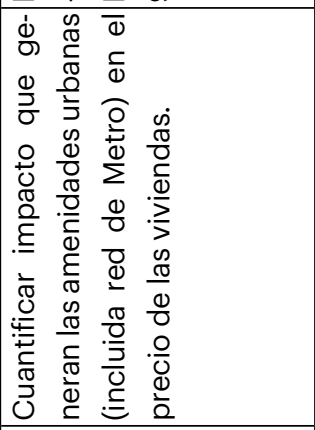 & 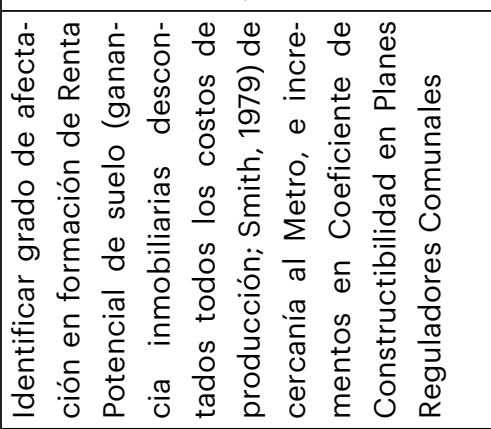 \\
\hline 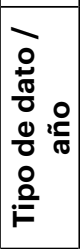 & 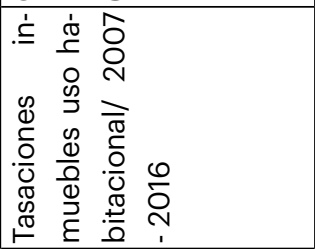 & 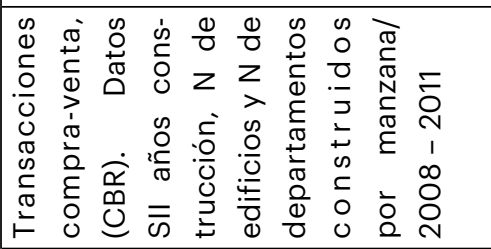 \\
\hline 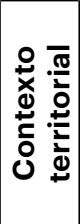 & 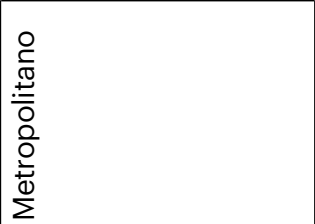 & 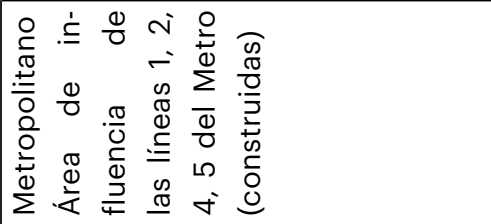 \\
\hline 号 & $\overline{\mathrm{D}}$ & 吕 \\
\hline 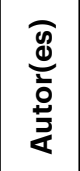 & 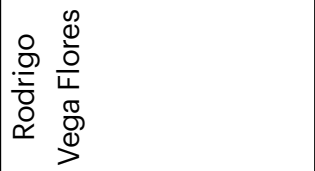 & 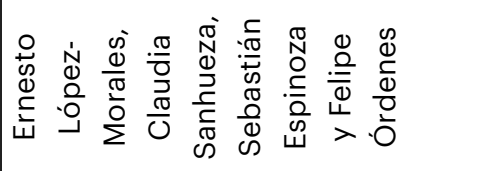 \\
\hline$\stackrel{ }{\stackrel{2}{2}}$ & 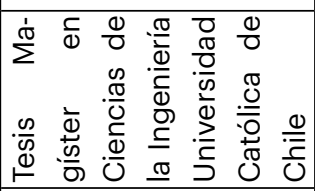 & 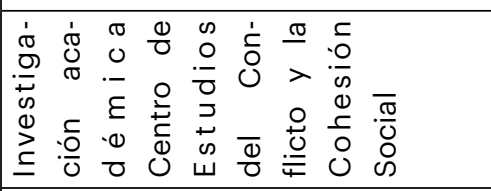 \\
\hline $\begin{array}{l}\frac{0}{2} \\
\stackrel{2}{E}\end{array}$ & 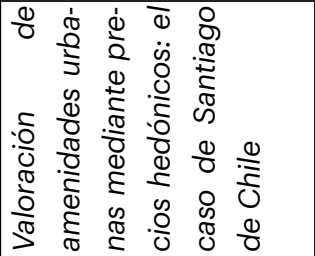 & 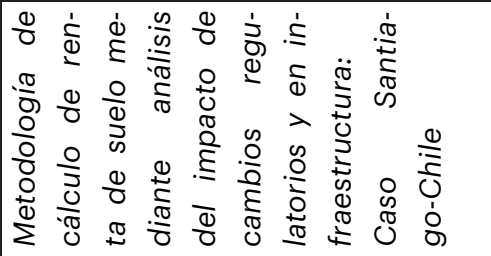 \\
\hline
\end{tabular}


La búsqueda de las investigaciones mencionadas, se centró inicialmente en la exploración de repositorios universitarios, teniendo como principal criterio de selección investigaciones con enfoque cuantitativo y de reciente elaboración. Posteriormente, se exploraron publicaciones académicas en internet, así como citas derivadas de la bibliografía general consultada y recomendaciones bibliográficas realizadas por los expertos entrevistados. Las siete investigaciones fueron analizadas y comparadas entre sí, cotejando el tipo de datos aportados, el año de levantamiento de éstos, y la escala del análisis de cada investigación, para finalmente elegir cinco investigaciones que pudieran ser complementarias y comparables entre sí.

El estudio de las fuentes citadas se centró en la síntesis y comparación de hallazgos a partir de los siguientes descriptores: nombre del estudio, año de elaboración o publicación, localización del estudio, tipo y fuente de los datos empleados, periodo estudiado y resultados. A partir de la disección y comparación de los contenidos, se analizaron los aportes y limitaciones de cada estudio y su aplicación en la investigación, identificando las fuentes más afines, con base en los siguientes criterios: (1) concurrencia de escalas de análisis metropolitana y local; (2) similitud en la temporalidad de los datos estudiados; (3) compatibilidad de los tipos de datos; (4) compatibilidad de la metodología con el tiempo y alcance de la investigación; (5) complementariedad en el estudio de la valorización ex ante / ex post a la construcción del Metro.

Adicionalmente, empleando un sistema de información geográfica (ArcMap 10), se representó y analizó la información georreferenciada extraída de las bases de datos de permisos de edificación (INE, 2017) y de López-Morales et al. (2019). La figura No 1 es la representación de proyectos inmobiliarios georreferenciados seleccionados con los siguientes criterios: (1) año de expedición entre 2010 - 2016; (2) número de pisos de la edificación mayor a 6 pisos; (3) destino residencial. Ello posibilitó la interpretación, generación y expresión cartográfica de la concentración de proyectos inmobiliarios en relación al trazado del Metro de Santiago. Para las figuras $\mathrm{N}^{\circ} 2$ y No3 se analizaron los datos provenientes de López-Morales et al. (2019) utilizados para calcular el aporte porcentual de infraestructura de Metro y regulaciones urbanas en la renta del suelo. Los autores construyeron una base de datos con 36.911 transacciones inmobiliarias entre los años 2008 y 2011 que permitió estimar el incremento en la ganancia de los propietarios particulares (RCS-1) y en la ganancia de las inmobiliarias (RCS-2). Para la construcción de la cartografía, se aislaron los datos correspondientes a la RCS-1 y RCS-2 y se aplicó el método cartográfico de interpolación de datos denominado Distancia Inversa Ponderada (IDW por sus siglas en inglés). En el método IDW, los puntos de muestreo se ponderan durante la interpolación de tal manera que la influencia de un punto en relación con otros disminuye con la distancia desde el punto desconocido que se desea crear. El resultado fue la concentración geográfica de los mayores valores encontrados para la RCS-1 y la RCS-2, variables cuya definición conceptual se expone a continuación.

\section{Las rentas de suelo rural y urbano}

La teoría de la renta de suelo se compone de tres ejes conceptuales: primero, el de la Renta de la tierra; segundo, el de la Renta del suelo urbano como reformulación de la renta de la tierra en el ámbito urbano; y tercero, el de la recuperación y redistribución de plusvalía urbana, que es propiamente la materia principal de este artículo. 
Respecto a lo primero, Marx (1995), basado en Adam Smith y David Ricardo, observó el fenómeno de la renta de la tierra y con ello sentó las bases para comprensión de las dinámicas económicas en torno al proceso productivo que se genera sobre éste. En su definición, Marx plantea que la tierra adquiere valor y genera renta en función de su transacción como mercancía, aun cuando no es un producto y por lo tanto no emplea medios ni tiempo de trabajo. La discusión ricardiana y marxista sobre el origen y la apropiación de las rentas de la tierra presenta un punto de inflexión en la incorporación que realiza Marx de la figura de la propiedad privada y el poder jurídico ejercido por la clase terrateniente, como principal factor determinante en la generación y acumulación de la renta de la tierra. El terrateniente, descansando en su condición de propietario, está capacitado para exigir una parte de la ganancia, por concepto de permitir el acceso y explotación de la tierra por parte del capitalista. De esta manera, la tierra adquiere cualidades como medio de producción y objeto de intercambio y la posibilidad de ser monopolizada mediante la apropiación individual. Esta condición de acumulación por parte del terrateniente y de necesidad de explotación por parte del capitalista, genera el precio del suelo. Como explica Jaramillo (2008:6), "lo que se transa en los negocios de terrenos, más que la tierra misma, es el derecho a percibir una renta", y es la magnitud de esta renta, la que determina el precio de la tierra en el mercado. Como se ve, la renta no se trata de un "plusvalor" o "plusvalía", ya que ésta última es la ganancia resultante de la remuneración incompleta pagada al obrero en la producción de un bien (apropiada por el capitalista sin realizar trabajo).

Respecto a lo segundo, los tipos de renta identificados por Marx (Rentas Diferenciales tipos 1 y 2, Renta Absoluta y Renta de Monopolio) son reformulados en el contexto urbano esclareciendo su rol en los procesos productivos inmobiliarios urbanos actuales. Por ejemplo, tales definiciones se cruzan con lo planteado por Smith (2012), para quien la producción de la renta del suelo urbano es realizada en dos tiempos. Un primer momento donde existe una notable diferencia de valor entre una Renta Potencial del Suelo (RPS) en aumento constante producto de normas públicas de construcción e inversiones públicas (y en menor medida privadas) en determinados sectores de la ciudad, y una Renta Capitalizada del Suelo (RCS), de menor valor presente, internalizable por los propietarios de suelo que por diversas razones no han realizado inversiones importantes sobre ese suelo. Eso es lo que se denomina como rent gap (brecha de renta). Luego, un segundo momento en el que, mediante inversión en la materia construida de suelo en su máxima intensidad posible, la RCS alcanza la RPS, con lo cual la rent gap se "cierra" (López-Morales, 2015). La renta maximizada a su nivel potencial finalmente es capitalizada por quien ahora ostenta la propiedad del suelo (la inmobiliaria, el nuevo propietario, o el antiguo propietario si tuvo capacidad para invertir en renovar la edificación), mediante el cobro de alquiler de la propiedad, o en una operación de compraventa, apareciendo como parte del precio final.

Sin embargo, se entiende que la maximización de la renta potencial resulta de la valorización del producto inmobiliario por la acción de factores externos ajenos a la voluntad del propietario y, por ende, teóricamente, se trata de un valor que debiese retornar al agente que lo genera, es decir, al Estado. Las causas de la valorización de la renta de suelo y la justificación de su recuperación pública, son clarificadas a continuación, existiendo coincidencia en el rol del Metro como principal fuente de maximización de la renta de suelo en la ciudad de Santiago. 


\section{Propuestas de cálculo de plusvalías por el Metro en Santiago}

En la ciudad de Santiago, la red de Metro constituye la inversión en infraestructura de transporte público más relevante, contando al momento de escribir este artículo (2019) con siete líneas en funcionamiento (líneas 1, 2, 3, 4, 4a, 5 y 6), tres líneas proyectadas (líneas 7, 8 y 9) y extensiones proyectadas (líneas 2,3 y 4 ).

López-Morales et al. (2019), destacan que además de los impactos de la red de Metro sobre la optimización de las condiciones de conectividad de los usuarios y la disminución de los tiempos de viaje, el Metro de Santiago se presenta como el "principal factor generador de valorización de ganancia inmobiliaria en la ciudad" (Renta Potencial de Suelo, o RCS-2). Esta ganancia no se distribuye homogéneamente en el territorio y se acentúa o disminuye en la medida en que los proyectos inmobiliarios se localizan a una distancia de un kilómetro a la redonda de las estaciones; radio en el que los proyectos inmobiliarios son vendidos a mayor precio. No obstante, los desarrolladores no pagan un precio (o Renta Capitalizada de Suelo, o RCS-1) proporcionalmente más alto por el suelo donde emplazan sus edificios. Como describen López-Morales et al. (2019), las empresas inmobiliarias, capturan casi en su totalidad, las plusvalías que se generan por la capitalización de la localización de sus proyectos en torno a la red de Metro, en un proceso iniciado por la compra y explotación del suelo cercano a las estaciones, mediante la construcción en altura y posterior venta de viviendas y oficinas a precios elevados. La fijación de un alto precio de venta, apoyada por la cercanía a bienes públicos, genera ganancias a sus inversores sin que se exija por parte del Estado, el retorno total o parcial de la valorización impulsada por su acción. El cálculo que establecen para la renta inmobiliaria es la ecuación residual que se obtiene restando al precio de venta $\left(P_{i c t}\right)$, el costo del suelo $\left(C S_{i c t}\right)$ y el costo de construcción $\left(C C_{i c t}\right)$ asociados al departamento i en la comuna $c$ en el tiempo $t$. La variable " $r$ " es la renta percibida por las inmobiliarias, en la siguiente ecuación: $r_{i c t}=P_{i c t}-C S_{i c t}-C C_{i c t}$.

Por otra parte, la ejecución del mayor y mejor aprovechamiento del suelo, encuentra en la regulación municipal muchas posibilidades y pocas restricciones para los desarrolladores. De acuerdo con López-Morales et al. (2014), los instrumentos de regulación de suelo operan en los municipios con el fin de atraer actividad inmobiliaria, maximizando el potencial de ganancia privada. La normativa urbana vigente, consignada en los Planes Reguladores Comunales (PRC) del Gran Santiago, está llena de normativas que permiten la máxima altura de las edificaciones, principalmente en zonas cercanas a la red de Metro.

Como ejemplo de esta relación entre la construcción en altura, las normas que la posibilitan y la red de Metro, se presenta en la Figura $\mathrm{N}^{0} 1$ la distribución de los permisos de edificación en altura con destino residencial. El resultado, es la distribución de proyectos de densificación en la ciudad, con especial énfasis en aquellos localizados en el área de influencia directa de la red de Metro (trazado y estaciones). Como es posible observar, el área de influencia de las estaciones de Metro, acoge la mayoría de permisos de construcción, evidenciando en el territorio la preferencia por los suelos cercanos a la red de transporte público para construir proyectos de vivienda en altura. 
La georreferenciación de los permisos de construcción para vivienda en altura, permite observar el patrón de distribución en el territorio de aquellas edificaciones inferiores a los 13 pisos, entre 13 y 20 pisos, y las que sobrepasan los 20 pisos. Estas alturas son posibilitadas en su ejecución por normas específicas contenidas en los Planes Reguladores Comunales (PRC) que permiten alturas libres sobre los principales ejes estructurantes del territorio comunal. Cabe destacar que muchos PRC que anteriormente permitieron altura de edificación libre, recientemente han modificado su normativa urbana en pro de la reducción de alturas. Si bien estas medidas mitigan parcialmente los efectos de la densificación desmedida en el territorio, continúan sin articular instrumentos de gestión que signifiquen el retorno de la plusvalía urbana adquirida por el máximo aprovechamiento normativo que se permite.

Figura № 1.

LOCALIZACIÓN DE PERMISOS DE EDIFICACIÓN PARA USO RESIDENCIAL, AÑOS 2010 - 2016. EDIFICIOS DE MÁS DE 6 PISOS.

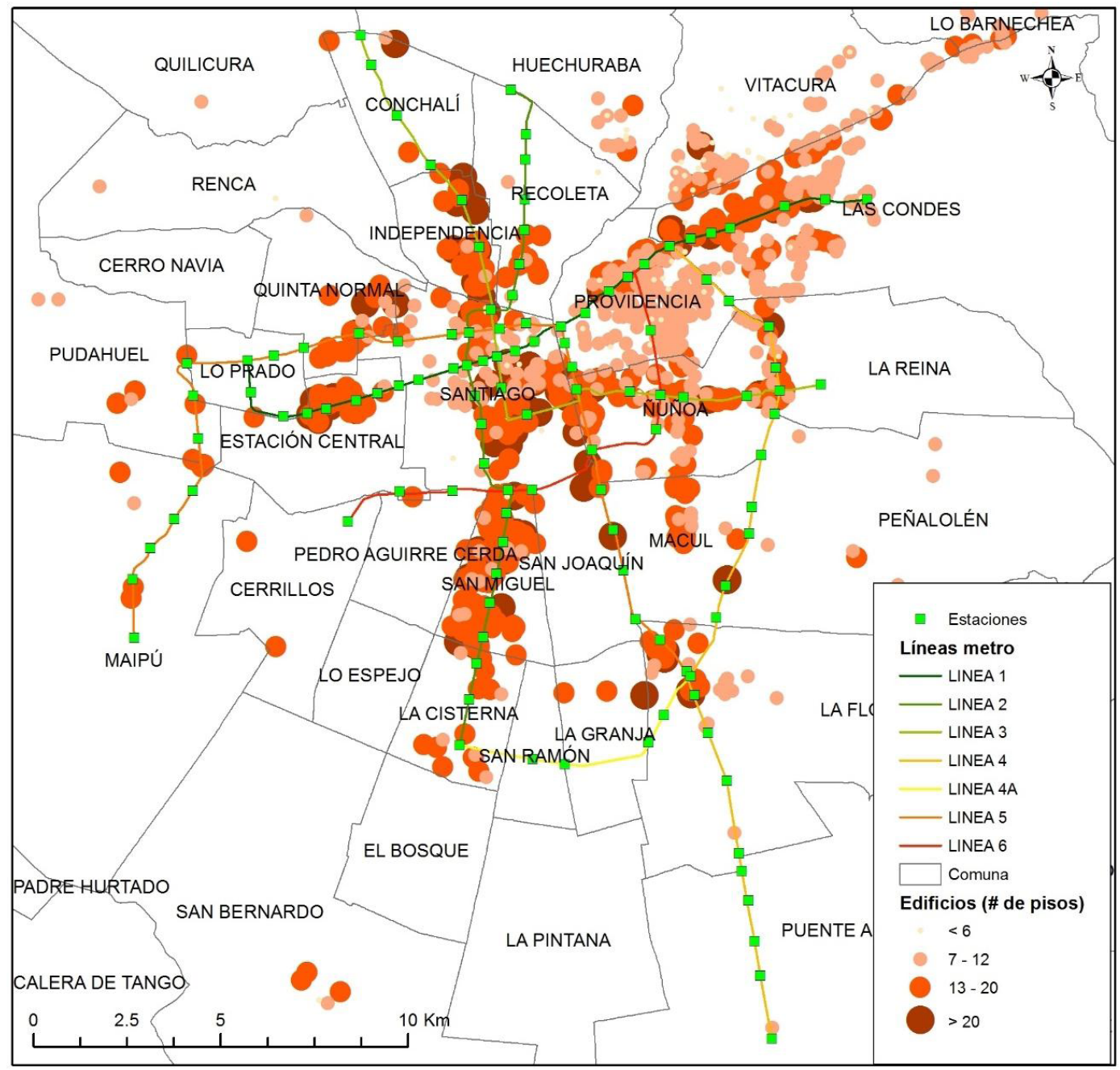

Fuente. Elaboración propia con datos georreferenciados de permisos de edificación INE (2017) 
La capitalización de plusvalías por anuncio y construcción de la red de Metro, por parte de empresas inmobiliarias, ha sido también objeto de investigación por parte de académicos y consultores, aportando sólida evidencia cuantitativa de la valorización que genera el Metro de Santiago. Para efectos de esta investigación, se analizaron comparativamente los datos extraídos de investigaciones realizadas entre los años 2014 y 2017 (Cuadro No1). Las conclusiones generadas se presentan a continuación:

\section{a) Variación en la valorización por cercanía a la red de Metro}

Los efectos máximos y medios derivado de la proximidad medida entre los 10 y los 500 metros a la redonda de amenidades urbanas como el trazado de Metro en superficie o elevado, reporta en la tasación del inmueble una variación máxima y media de $-14,3 \%$ y $-3,5 \%$ respectivamente (lo que habla del impacto negativo del viaducto elevado en su entorno circundante), situación que cambia cuando se observa la variación máxima y media de tasaciones próximas a estaciones de la red de Metro, que van entre de 19,4\% y 4,3\% (Vega, 2017). Este porcentaje máximo de variación, es cercano al estimado por López-Morales et al. (2019) quienes establecen un aumento en la valorización de inmuebles reportado en transacciones de bienes raíces y una ganancia inmobiliaria cercana al $25 \%$ derivada de la materialización de esfuerzos públicos en inversión de transporte. Por otro lado, Transsa \& Atisba (2014) encuentran un promedio de captación de plusvalía $21 \%$ y $22 \%$ para áreas de influencia de estaciones de Metro entre los 300 y 600 metros de distancia respectivamente. Los tres estudios, pese a estar basados en diferentes tipos de datos, comprueban que existe una acción de valorización y de capitalización privada de una amenidad urbana como es el Metro. Se observa que el incremento en el valor del suelo reportado en tasaciones y transacciones de bienes raíces se encuentra entre el $19,4 \%$ y el $25 \%$, a razón de la proximidad entre el inmueble y la estación. La renta potencial en estas zonas, las hace atractivas para el desarrollo inmobiliario, que colma las áreas cercanas a la red de Metro en espera de capitalizar la plusvalía que le genera, mediante la construcción de vivienda en altura.

\section{b) Red de Metro construida}

Para López-Morales et al. (2019), las líneas de Metro 1, 2, 4, y 5, en funcionamiento para los años de los datos empleados por el estudio (2008-2011), aumentan significativamente la valorización del suelo representando ganancias inmobiliarias diferentes por comuna. Esta valorización del suelo varía entre los 19,7\% para Providencia, un 22\% para las comunas de La Cisterna, La Granja y La Florida, un $48 \%$ para Santiago Centro y un $52 \%$ para la comuna de Las Condes. No obstante, la valorización que genera el funcionamiento del Metro a los propietarios originales del suelo es de solo un 5,5\%. Los autores plantean que estos porcentajes de valorización extraídos de operaciones de compraventa, contradicen la noción de que el propietario original, cuando es persona natural, es el principal capitalizador de la instalación de la red de Metro, pues las ganancias inmobiliarias derivadas de la explotación del suelo a razón de su localización y potencial de constructibilidad, son significativamente mayores y solo son capturadas por agentes inmobiliarios privados.

La Figura $\mathrm{N}^{\circ} 2$ realizada a partir de la base de datos levantada por López-Morales et al. (2019) muestra precisamente cómo las rentas de suelo percibidas por transacciones de predios sin inversión reciente (casas, galpones, eriazos) tienen una distribución relativamente pareja en la 
ciudad, con excepción de su notablemente mayor valorización concentrada en la zona oriente del Área Metropolitana, donde tradicionalmente se encuentran las viviendas de mayor precio, tamaño y calidad, y sectores muy puntuales de las áreas con mayor valorización en el centro de Santiago (Santa Lucía, Lastarria y rivera del Río Mapocho). Sin embargo, la Figura Nº3 da cuenta de mayor distribución territorial de las rentas potenciales capturadas por inmobiliarias, que alcanzan su mayor valorización (25\% adicional promedio) por estar localizadas dentro del radio de $1 \mathrm{~km}$ alrededor de alguna estación de Metro, que estando fuera de éste. Si bien la Figura 3 da cuenta también de zonas de intensa valorización de la renta inmobiliaria fuera del área de influencia del Metro (zona oriente) la correlación con esta red queda bastante evidenciada.

Figura $N^{\circ} 2$.

RENTA PERCIBIDA POR PROPIETARIOS ORIGINALES (RCS-1)

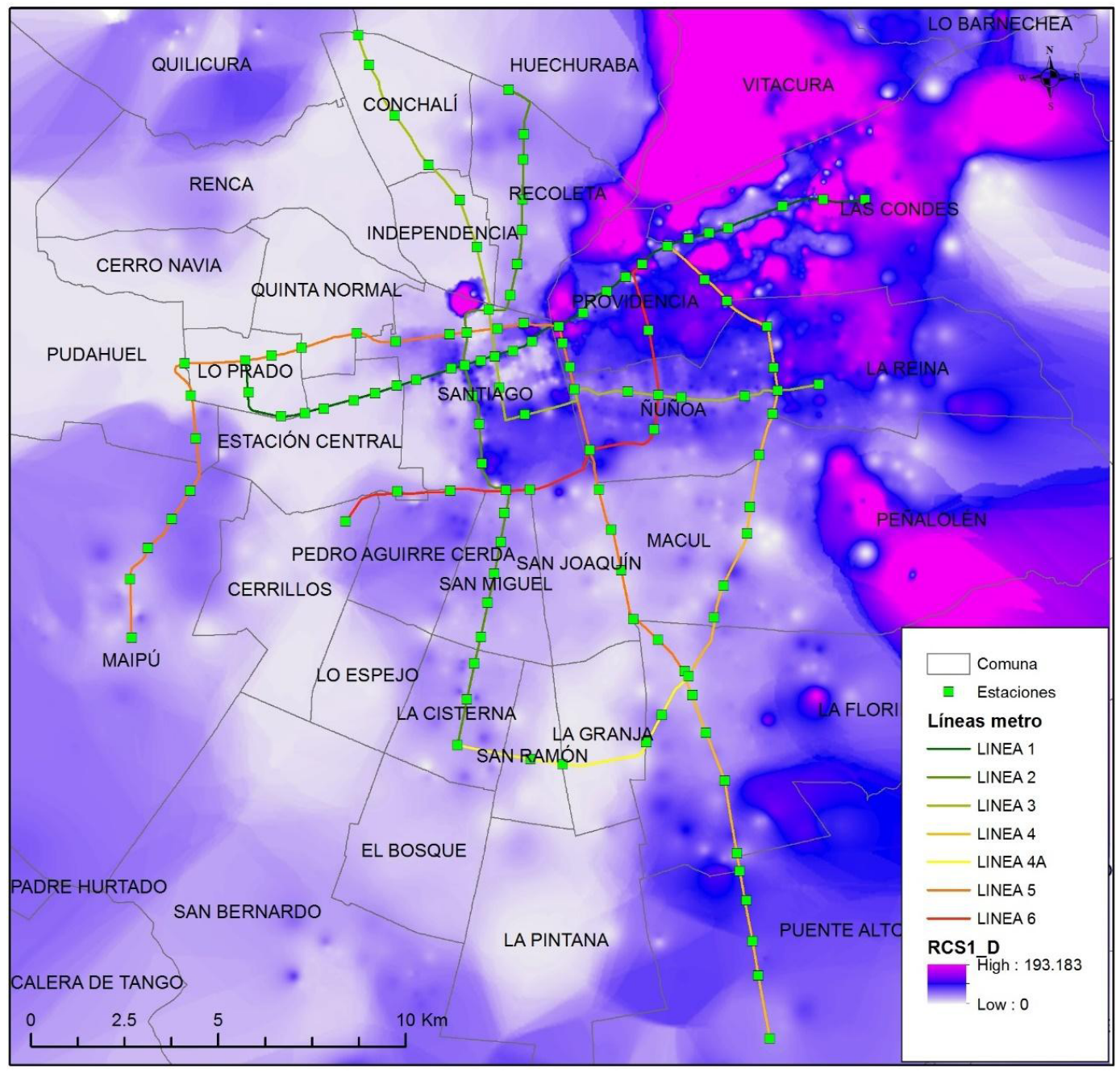

Fuente. Elaboración propia con datos georreferenciados de López-Morales et al. (2019) 
Figura No 3.

RENTA PERCIBIDA POR DESARROLLADORES INMOBILIARIOS (RCS-2)

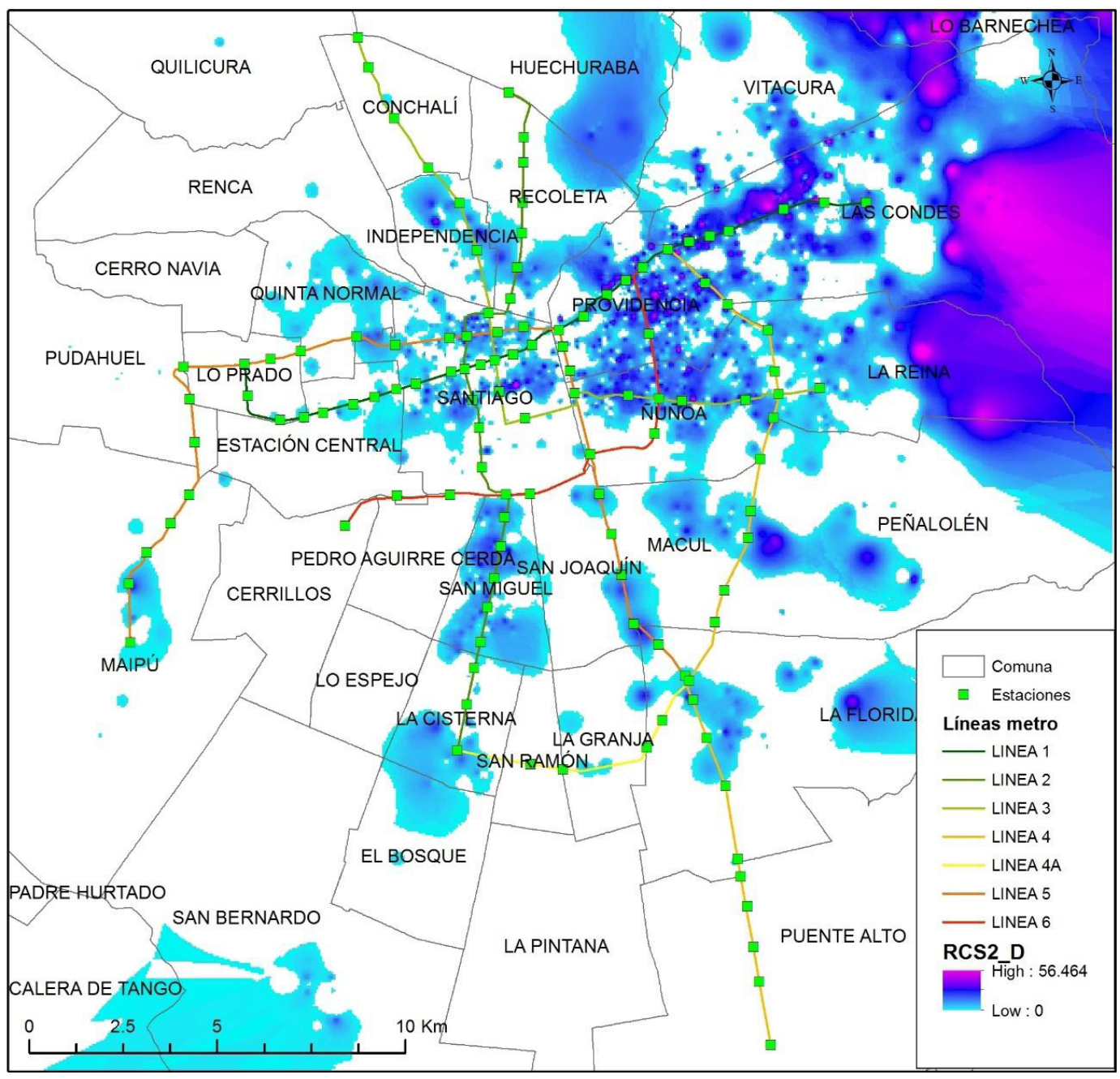

Fuente. Elaboración propia con datos georreferenciados de López-Morales et al. (2019)

\section{c) Red de Metro proyectada}

Las consultoras inmobiliarias Transsa \& Atisba (2014), en un ejercicio conjunto realizado en el área de influencia de las líneas de Metro proyectadas y en construcción a la fecha del estudio (líneas 3 y 6 ), formulan que el anuncio del proyecto de construcción del Metro, produce un aumento en el valor comercial de inmuebles tasados que varía en función del uso que se instale. Esto lo identifican tanto para construcciones como para terrenos. Así, para el uso habitacional, el anuncio genera una valorización del $21 \%$ en las áreas de influencia directa de estaciones (300 metros) y de un $20 \%$ en los tramos comprendidos entre estaciones. Al respecto, cabe aclarar que el estudio en mención, no hace diferencia entre inmuebles residenciales tipo departamento y tipo casa. La valorización del inmueble tipo departamento es capitalizada por agentes inmobiliarios 
en la transacción comercial, mientras que la valorización de inmuebles tipo casa, remite al valor del suelo y a su potencial edificatorio, mismo que es aprovechado por agentes inmobiliarios que compran predios y construyen el máximo aprovechamiento normativo.

Para los usos comerciales, la plusvalía que se genera en inmediaciones de las estaciones de Metro es más notoria, pues "estos usos pagan más por el suelo y tienden a privilegiar la proximidad con la estación para capturar el flujo de población flotante, que es clave en la venta del retail." (Atisba \& Transsa, 2014:10). Los aumentos de valor reportados para usos comerciales alcanzan un $27 \%$ en las áreas de influencia directa (300 metros). El estudio de las investigaciones citadas deja ver que existen dos ámbitos de la capitalización de la localización de la red de Metro:

El primero corresponde a la capitalización por parte de un propietario original, que en la mayoría de casos es una persona natural, que no ha ejecutado el máximo posible de la norma urbanística de su predio y cuya valorización se ve registrada en el avalúo catastral efectuado por el Servicio de Impuestos Internos para el cobro del impuesto territorial. De ser perfeccionado, este impuesto actuaría en este escenario específico como captador parcial y proporcional de plusvalía.

El segundo ámbito corresponde a la capitalización que realiza el sector inmobiliario en una etapa posterior a la adquisición del precio. En este punto, se construye el aprovechamiento máximo que dicta la norma urbanística para el predio específico, generando unidades de vivienda, comercio y/o servicios que son vendidas en un precio máximo fijado por el mercado y que representa una enorme ganancia para el operador inmobiliario. Actualmente, la Ley de Aportes al Espacio Público prevé la mitigación de las externalidades producidas por los proyectos de densificación en una comuna, pero esta mitigación está lejos de tratarse de una recuperación de plusvalía. Por esta razón, es relevante formular mecanismos que logren recuperar parcialmente las ganancias inmobiliarias generadas por una combinación entre las normas urbanísticas contenidas instrumentos regulatorios, que promueven máximos aprovechamientos del suelo urbano y la construcción de obras públicas de infraestructura de transporte que viabiliza esta densificación.

\section{Principios de recuperación, redistribución y regresividad de la "plusvalía" urbana}

Desde la base teórica de Henry George, y su obra "Progreso y Miseria. Indagación acerca de la causa de las crisis económicas y del aumento de la pobreza con el aumento de la riqueza" (1879), se pueden extraer tres aportes directamente atingentes a esta investigación. George ([1879] 2004:103) destaca la diferencia fundamental que existe entre la propiedad de las cosas que son el producto del trabajo y la propiedad en la tierra, al afirmar que "nadie puede tener derecho a la propiedad de algo que no sea producto del trabajo propio o del trabajo de quien le haya cedido su derecho". Como consecuencia, y dado que ningún individuo había "producido" la tierra, ésta era propiedad de la comunidad, de todos los hombres (Ramos, 2001). George reconoce, al igual que Marx, una clase social rentista apropiada de la riqueza a razón de ostentar la propiedad jurídica de la tierra. La propuesta de George consistió en hacer común la propiedad de la tierra, valiéndose 
de herramientas que no implicaban confiscación o desposesión a sus propietarios jurídicos, definiendo así, el Impuesto Único de la tierra.

Desde América Latina, las contribuciones a esta discusión conceptual no han sido pocas. Para Jaramillo (2001:71), la plusvalía urbana es la "expresión paradigmática de una ventaja privada y unilateral que emerge de un proceso colectivo, la urbanización", por lo que es imperativa su recuperación total o parcial, cumpliendo así el ciclo de valorización, recuperación de plusvalía y redistribución de la inversión pública. Es precisamente esta acción de retorno de los beneficios y redistribución la que debe ser ejercida equitativamente para garantizar el acceso colectivo a los bienes urbanos que se financian. Más recientemente, Furtado $(1997 ; 2000)$ y Furtado \& Smolka (2001; 2014) han introducido el énfasis en el principio ético de la justicia redistributiva de las intervenciones urbanas que son financiadas por la recuperación de plusvalía. Sin embargo, es conveniente decir también que la recuperación de plusvalías urbanas no garantiza por sí misma la disminución de desigualdades socio espaciales acumuladas (Furtado 1997), siendo necesaria la implementación de mecanismos de inversión en zonas que no pueden depender para su desarrollo de la recuperación de plusvalías urbanas: zonas por lo general carenciadas, exentas de impuestos y contribuciones y que al mismo tiempo no presentan posibilidades reales para el anclaje de capital inmobiliario que pueda aprovechar las condiciones de edificación y hacer aportes sobre las rentas que produce.

El entendimiento de las acciones de distribución y redistribución plantea la necesidad de separar los beneficios espaciales de las necesidades básicas (Furtado, 2000), pues la acumulación de beneficios urbanos en zonas de altos ingresos, no debería ocurrir antes de que las zonas deficitarias de la ciudad cuenten con acceso a la infraestructura urbana de servicios básicos. Por consiguiente, si bien la redistribución desde áreas ricas hacia áreas pobres implica la implementación de mecanismos de transferencia de aprovechamientos urbanos "como una alternativa para satisfacer las necesidades urgentes de las zonas pobres, en combinación con oportunidades y demandas específicas de las zonas pudientes", la estimulación de los incrementos de valor del suelo en zonas de alta renta, termina por aumentar la brecha entre zonas ricas y zonas pobres de la misma ciudad (Furtado, 2000: 234).

\section{Chile: avances recientes en recuperación de rentas de suelo}

Al año 2018, los avances recientes en el marco de la legislación y las políticas públicas urbanas se centran en la aprobación en los dos últimos años de la "Ley de Aportes al Espacio Público" (LAEP, 2016) y la "Ley de Transparencia del Mercado del Suelo e Incrementos de Valor por Ampliaciones del Límite Urbano" (LTMS, 2018). Ambas políticas han sido vistas como potenciales aportes a un régimen de captura de plusvalías urbanas con efectos positivos en redistribución. Sin embargo, como muestra la evidencia, ello está lejos de ocurrir.

La Ley 20.958 de Aportes al Espacio Público (LAEP), establece un sistema que propende principalmente por la mitigación de los impactos que la actividad inmobiliaria pueda tener sobre la infraestructura de movilidad y el espacio público instalados en determinada zona de la ciudad. Así, la ley queda compuesta por dos partes: la mitigación, como una versión evolucionada del 
Estudio de Impacto sobre el Sistema de Transporte Urbano -EISTU-, y los aportes, directamente relacionados con la dotación local de espacio público.

Dos puntos principales llaman la atención en el estudio de la LAEP en relación con la gestión del desarrollo urbano y la recuperación de plusvalía. Por un lado, la proporción en los aportes para mejoramiento de la infraestructura vial de mínimo 70\%, frente a un máximo de $30 \%$ para espacio público ${ }^{10}$, no se condice con la necesidad de crear más y mejores espacios públicos. Esto, sumado al cálculo de aportes mediante la ejecución de un avalúo fiscal y no comercial, derivaría en aportes reducidos en suelo y/o dinero, aprovechables en la construcción local de obras públicas. Otro punto destacable es su prácticamente nula capacidad de redistribución, al confinar su aplicación al ámbito territorial de la comuna, lo cual es gravitante siendo Santiago una metrópolis con altos niveles de disparidad de ingresos entre comunas. Cabe señalar que si bien los aportes pueden financiar obras de espacio público intercomunal'11, entre unidades territoriales vecinas, no contempla la posibilidad de redistribución en una escala metropolitana. Asimismo, la mencionada Ley excluye por completo la destinación de los recursos recolectados al financiamiento de la construcción de vivienda de interés social en la comuna, algo que sí ocurre en la realidad brasilera con las "cargas onerosas" y en Colombia con los porcentajes mínimos de vivienda social incluidos como obligaciones urbanísticas.

Por otro lado, la Ley de Transparencia del Mercado del Suelo e Incrementos de Valor por Ampliaciones del Límite Urbano, en adelante LTMS, se formula atendiendo dos problemas fundamentales; por un lado, la falta de transparencia en la información de las transacciones de compraventa de predios urbanos; y por otro, las enormes ganancias producidas por la venta de terrenos recalificados, es decir, aquellos que se integran al límite urbano en su expansión. Para esto, propone establecer un impuesto que permita al Estado capturar una porción del incremento del valor producto de aquellos procesos de cambio normativo donde se amplía el límite urbano. La tasa propuesta para gravar estos aumentos, es del 10\% cuyo cobro se materializará en el momento de la transacción y que se distribuye en dos partes: un 37,5\% que se incorporará al patrimonio de la municipalidad donde se localice el respectivo bien raíz, y será empleado en el financiamiento de obras de desarrollo local; y un 62,5\% se incorporará al Fondo Común Municipal (FCM). Esta partida asignada al FCM, puede ser considerada como una acción de redistribución de la plusvalía recuperada por la aplicación del impuesto.

Sin embargo, tres aspectos concentran los principales acuerdos y disensos sobre la LTMS y lo que derive de su aplicación: (1) la tasa del 10\% representa una porción marginal de las ganancias obtenidas en las transacciones sobre terrenos antes rurales que pasan a ser urbanos; (2) la exclusión de las plusvalías que se generan en actos normativos diferentes a la ampliación del límite urbano, por ejemplo las generadas por obras públicas; (3) la necesidad de ejecutar reavalúos periódicos para estimar el incremento en el valor del suelo de expansión urbana, perfeccionando el proceso actual de avalúo llevado a cabo por el Servicio de Impuestos Internos. Este perfeccionamiento pasa por desligar la periodicidad de los avalúos de los ciclos políticos, y acercar el avalúo fiscal a valores establecidos por el avalúo comercial, pues el primero se encuentra muy por debajo de los precios en los que son transados los inmuebles.

Ley No. 20.958 Establece un sistema de aportes al espacio público. Artículo 180

Ley No. 20.958 Establece un sistema de aportes al espacio público. Artículo 180 
Particularmente, la LAEP está lejos de constituir un mecanismo de captura y mucho menos redistribución de plusvalía, y se formula más bien como mecanismo de mitigación de las externalidades ocasionadas por proyectos de densificación, requiriendo la realización de aportes en suelo o en dinero en la comuna donde se localiza el proyecto inmobiliario. Es más, los expertos entrevistados argumentan que el bajo porcentaje de aporte obligado y la disposición de los recursos en una relación de 70\% para infraestructura de movilidad y 30\% para obras de espacio público, lo convierten en un mero instrumento de mitigación.

Bajo la LAEP, se configurarán tres tipos de comunas:

1. Con intenso desarrollo inmobiliario, es decir, las de mejor dotación de bienes públicos y acceso a la Red de Metro, llamadas a recaudar los aportes y reinvertirlos al interior de la misma unidad administrativa, efectuando mejoras en la red vial y en el espacio público y generando con esto, valorización de suelo adicional.

2. Sin desarrollo inmobiliario, pero con acceso al Metro, que podrán ver en la aplicación de la LAEP la posibilidad de aumentar el ingreso municipal para financiar obras públicas y con ello podrían patrocinar la flexibilización de sus normas urbanísticas para propiciar la llegada del desarrollo inmobiliario que posibilita la activación del proceso de recaudo e inversión de los aportes.

3. No atractivas para el desarrollo inmobiliario, por su baja accesibilidad a la red de Metro o falta de dotación de bienes públicos, que no participarán en el recaudo directo de los aportes ni contarán con esta vía para financiar mejoramiento de vialidad y espacio público.

Esto quiere decir que la LAEP corre el riesgo de convertirse en un mecanismo regresivo que acentúe las diferencias e inequidades dotacionales entre zonas de Santiago. Esta investigación considera que esta ley sienta un precedente para la participación del Estado en las plusvalías apropiadas por el sector inmobiliario en Chile, pero está lejos de ser un instrumento que cumpla con acciones de recuperación y redistribución, al no basarse en la valorización del suelo y al restringido alcance local de su aplicación que posibilita efectos regresivos.

Por otro lado, la LTMS, pretende gravar los incrementos en el valor del suelo originados en la recalificación de suelo de rural a urbano. Su recaudo y gestión son también de escala local, dejando un gran número de comunas que por su situación geográfica, al no compartir límites con el suelo rural, no pueden participar de los recursos que se generan en su aplicación.

\section{Conclusiones}

En América Latina, la recuperación de plusvalía posibilitada por la valorización del suelo a través de la acción planificadora del Estado, es aprovechada por agentes privados quienes capitalizan las ventajas normativas e infraestructura instalada, a cambio de escasas obligaciones que implicarían el retorno a la comunidad de las rentas de suelo excedentes. 
La red de Metro de Santiago ejemplifica como solamente el anuncio y construcción de obras de infraestructura ya genera aumento en los valores del suelo, mismo que es capitalizado por dos tipos de agentes privados: el propietario original y el desarrollador inmobiliario. El primer agente, no ejecuta al máximo la norma urbanística que corresponde a su predio y la valorización de este es registrada en el avalúo catastral (un impuesto territorial con base suelo, que perfeccionado y robusto, captaría proporcionalmente esta valorización). El segundo agente, el desarrollador, maximiza el aprovechamiento de la norma urbanística otorgada, densificando y produciendo bienes inmobiliarios que son transados en el mercado al mayor precio posible, multiplicando las utilidades. Actualmente, la Ley de Aportes al Espacio Público pretende exigir a este desarrollador el pago de aportes y la internalización de las externalidades producidas por la densificación.

Sin embargo, ¿es el desarrollador inmobiliario quien debe asumir el pago de la plusvalía? La formación de las plusvalías urbanas, al tratarse de un proceso que implica la valorización del suelo en función de su localización y del rendimiento potencial de la normativa otorgada, conlleva la capitalización de los beneficios de cercanía a bienes urbanos de carácter público. Estos, por definición, son materializados, expandidos, renovados y mantenidos con recursos que gestiona el Estado y que representan el esfuerzo de la comunidad en general (más allá de los efectos de externalidades positivas adicionales que eventualmente se puedan generar entre esfuerzos privados). Contrariamente, las rentas generadas colectivamente, son apropiadas por desarrolladores privados que detentan la propiedad jurídica del suelo y los medios de producción para materializar su mayor y mejor aprovechamiento. Por lo tanto, quien en el ejercicio de maximización de las ventajas normativas y locativas, obtiene el mayor rendimiento, debe ser el objeto de aplicación de los instrumentos de recuperación de plusvalía de mayor envergadura y con mayor posibilidad de movilización de recursos.

Podrían existir algunas reticencias en contra de este principio elemental, por ejemplo aquéllas que usan como argumento que, con la recuperación de plusvalías, las inmobiliarias enfrentarán mayores costos y por ende aumentarán los precios de venta de la vivienda, con lo que la demanda se verá afectada. Sin embargo, se sabe que cuando los mercados son competitivos y una oferta diversificada de numerosos actores, ello asegura cierta elasticidad ante estas pérdidas. Son más importantes las posibilidades de que el Estado recupere estos recursos que ha invertido en el espacio urbano con el fin de, entre otros objetivos, proveer vivienda bien localizada en la ciudad que satisfaga la demanda residencial de amplios segmentos medios y bajos, actualmente no satisfecha por el mercado de vivienda.

Pero aún si la recuperación de plusvalía se efectuase, existiría en Chile, y gran parte de América Latina, un desbalance entre las dos acciones (recuperación y redistribución). Lo analizado en el contexto regional y chileno respalda esta afirmación: no existe un instrumental expresamente dedicado a la redistribución equitativa de los recursos recuperados. Es decir, está posibilitada la recuperación de la plusvalía, pero no así el efecto redistributivo. De este desbalance es posible concluir que instrumentos concebidos como equitativos, como las nuevas leyes chilenas en materia de gravar el desarrollo inmobiliario o incorporación de suelo rural a urbano, comportan efectos regresivos en el territorio. La instrumentalización de la recuperación de plusvalía solo como medio para financiar la política urbana municipal, deriva en la localización de la inversión pública en el mismo lugar donde fue generada la plusvalía recuperada. Se cumple entonces un ciclo regresivo compuesto por la valorización, la recuperación de plusvalía, la reinversión de recursos en 
el sitio y la revalorización de la zona que los recibe, siendo ésta a la vez generadora y beneficiaria. En Chile, aún resta avanzar en materializar instrumentos de captura de plusvalía de suelo urbano que sean efectivamente redistributivos.

\section{Referencias}

AGOSTINI, Claudio A.; PALMUCCI, Gastón. Capitalización heterogénea de un bien semipúblico: EI Metro de Santiago. Cuadernos de economía, 2008, vol. 45, no 131, p. 105-128.

ARRIAGADA LUCO, Camilo; SIMIONI, Daniela. Dinámica de valorización del suelo en el área metropolitana del Gran Santiago y desafíos del financiamiento urbano. CEPAL, 2001.

BLANCO, B., et al. El potencial de la captura de plusvalías para la financiación de proyectos urbanos: consideraciones metodológicas y casos prácticos. Andrés G. Blanco B./Nancy Moreno M./ David M. Vetter/Marcia F. Vetter. 2016.

BRASIL. Ley Federal N 10.257 Estatuto de la Ciudad, 2001

CARPIO DÍAZ, Guillermo. Precio del suelo y metodologías de avaluación. Exploración para la captura de plusvalías urbanas en el caso de la región metropolitana de Santiago de Chile. 2014.

CHILE. Ley No. 4180 Aprueba Proyecto de Pavimentación de Santiago, 1927

CHILE. Política Nacional de Desarrollo Urbano. Ministerio de Vivienda y Urbanismo, 2014

CHILE. Propuesta para una política de suelo para la integración social urbana. Consejo Nacional de Desarrollo Urbano del Gobierno de Chile. 2015

CHILE. Ley Nº 20.958 Establece un sistema de aportes al espacio público, 2016.

CHILE. Ley $N^{\circ} 21.078$ Sobre transparencia del mercado del suelo e impuesto al aumento de valor por ampliación del límite urbano, 2018

COLOMBIA. Constitución Política, 1991.

COLOMBIA. Ley 388 de desarrollo territorial, 1997

ECUADOR. Ley s/n Ley Orgánica de Ordenamiento Territorial, Uso y Gestión del Suelo, 2016.

FURTADO, Fernanda. Instrumentos para la recuperación de plusvalías en América Latina: Debilidad en la implementación, ambigüedad en la interpretación. Cambridge, Mass: Lincoln Institute of Land Policy, 1997. 
FURTADO, Fernanda. Reformulación de las políticas de recuperación de plusvalías en América Latina. Perspectivas urbanas. Temas críticos en políticas de suelo en América Latina. Ed. Lincoln Institute of Land Policy, Massachusetts (USA), 2007.

GEORGE, Henry. Progreso y miseria: investigación sobre la causa de las crisis industriales y del aumento de la pobreza con el incremento de la riqueza: el remedio. Casa Editorial Maucci, 1929.

INSTITUTO NACIONAL DE ESTADÍSTICAS DE CHILE. Georeferenciación de permisos de edificación nacional. Recuperado de http://www.ine.cl/herramientas/galeria-de-mapas/permisos-de-edificacion, 2017

JARAMILLO, Samuel. La experiencia colombiana en la recuperación estatal de los incrementos del precio del suelo. La Contribución de Valorización y la Participación en Plusvalías. eurelibro s, 2001, p. 71.

JARAMILLO GONZÁLEZ, Samuel. Hacia una teoría de la renta del suelo urbano. Ediciones Uniandes-Universidad de los Andes, 2008.

LÓPEZ, Ernesto; MEZA, Daniel; GASIC, Ivo. Neoliberalismo, regulación ad-hoc de suelo y gentrificación: el historial de la renovación urbana del sector Santa Isabel, Santiago. Revista de Geografía Norte Grande, 2014, no 58, p. 161-177.

LÓPEZ-MORALES, Ernesto. Assessing exclusionary displacement through rent gap analysis in the high-rise redevelopment of Santiago, Chile. Housing Studies, 2016, vol. 31, no 5, p. 540-559.

LÓPEZ-MORALES, Ernesto; SANHUEZA, Claudia; ESPINOZA, Sebastián; ÓRDENES, Felipe. Verticalización inmobiliaria y valorización de renta de suelo por infraestructura pública: un análisis econométrico del Gran Santiago, 2008-2011. EURE, 2019, vol. 45, no136, p. 113-134. DOI: $10.4067 /$ SO250-71612019000300113

MALDONADO, María M. Recuperación de plusvalías. Perspectivas urbanas. Temas críticos en políticas de suelo en América Latina. Ed. Lincoln Institute of Land Policy, Massachusetts (USA), 2007.

MARX, Karl. Capital: The process of capitalist production as a whole, ed. by Frederick Engels. Tr. from the 1st German ed. by Ernest Untermann. CH Kerr, 1909.

ORREGO, Carlos. Reavalúo nacional de propiedades año 2018. Recuperado de http://www.asatch. cl/reavaluo-nacional-de-propiedades-ano-2018/. Santiago, Chile, 2017.

RAMOS GOROSTIZA, José Luis. Henry George en la historia del pensamiento económico: razones para una revalorización. 2001.

RIVAS QUESADA, Javier Humberto. Uso del método de precios hedónicos, para estimar variación en precios de viviendas producto de nuevas líneas de Metro. 2015. 
SABATINI, Francisco; CÁCERES, Gonzalo. Santiago de Chile: la recuperación de plusvalías puesta en perspectiva histórica. Recopilación de plusvalías en América Latina", por Smolka M. Y Furtado F. (2001), LOM editores, Santiago de Chile, 2001.

SMITH, Neil. La nueva frontera urbana: ciudad revanchista y gentrificación. Traficantes de sueños, 2012.

SMOLKA, M.; FURTADO, Fernanda. Lecciones aprendidas de la experiencia de América Latina con la recuperación de plusvalías. Land Lines, julio, 2001, no 4.

SMOLKA, Martim; AMBORSKI, David. Recuperación de plusvalías para el desarrollo urbano: una comparación inter-americana. EURE (Santiago), 2003, vol. 29, no 88, p. 55-77.

SMOLKA, M. O.; FURTADO, F. Instrumentos notables de políticas de suelo en América Latina. Lincoln Institute of Land Policy. 2014.

SMOLKA, Martim. Implementación de la recuperación de plusvalías en América Latina. Políticas e instrumentos para el desarrollo urbano, Lincoln Institute of Land Policy, Cambridge, MA, 2014.

URUGUAY. Ley No 18.308 Ley de Ordenamiento Territorial y Desarrollo Sostenible, 2008.

TRANSSA, Consultores Inmobiliarios Ltda; ATISBA, Estudios y Proyectos Urbanos. Plusvalía potencial por anuncio de líneas 3 y 6 del Metro, 2014. Estudio preparado para el diario El Mercurio, Santiago de Chile.

TRIVELLI, Pablo. El suelo urbano, fundamento del hábitat y los negocios. Revista Igualdad y Democracia, 2015, no 3, p. 39-56

VEGA FLORES, Rodrigo Andrés. Valoración de amenidades urbanas mediante precios hedónicos: el caso de Santiago de Chile. 2017.

WAINER, Pablo; GASIC, Ivo; BAUZÁ, Marcelo. Grandes Promotores Inmobiliarios. Oferta y Mercado Objetivo. Santiago Metropolitano. 2016. Inciti. 